\title{
PROBABILISTIC INVESTIGATION ON DYNAMIC RESPONSE OF DECK SLABS OF HIGHWAY BRIDGES
}

\author{
Chul-Woo Kim \\ Research Scientist, Ph.D., Department of Civil Engineering, Kobe University, Japan \\ cwkim@kobe-u.ac.jp \\ Mitsuo Kawatani \\ Professor, Dr. Eng., Department of Civil Engineering, Kobe University, Japan \\ m-kawa@kobe-u.ac.jp
}

\begin{abstract}
Probabilistic assessment on the code-specified impact factors of RC decks is investigated by means of a three-dimensional traffic-induced dynamic response analysis of bridges combined with the Monte Carlo simulation technique. The random variables considered in the simulation are the roadway roughness, bump height, traveling position of vehicles, vehicle running speed and axle load of three-axle vehicles. Statistical parameters of the random variables are taken from surveying data on Hanshin and Meishin Expressways in Japan. A simple span steel-girder bridge with $\mathrm{RC}$ decks that is experimentally verified is considered as a numerical example. This study demonstrates that the impact factor of the deck near expansion joints dominates the design impact factor due to the bump at the expansion joint of bridges.
\end{abstract}

Keywords: traffic-induced vibration, RC deck, bump, impact factor, reliability assessment

\section{Introduction}

The performance of reinforced concrete (RC) decks of highway bridges mainly depends on cracking damages. Thus the rational criterion for the performance level of $\mathrm{RC}$ decks provides useful assessment tool for decision making related to the inspection, repair, upgrading and replacement of existing steel plate girder bridges based on life-cycle costs, since the 
$\mathrm{RC}$ deck, being directly subjected to wheel loads of vehicles, is more easily damaged than other structural members in steel highway bridges [5].

It is apparent that, except corrosion due to environmental factors, trucks or traffic loads play an important role in the deterioration of $\mathrm{RC}$ decks. Traffic loads are usually affected by the roadway roughness, dynamic properties of vehicles, vehicle speed, etc, and the dynamic effect is usually considered in design as the impact factor. For decks, moreover, a bump near expansion joints is another important factor because of the impulsive loading effect generated by vehicles passing over the bump [11] and [15].

Most of all the existing research topics related to the deck have been focused on static responses. Few research on dynamic responses of decks due to moving vehicles have been investigated, even though a fatigue problem of decks as a part of dynamic problems has been one of wide spreading research themes. Moreover, in civil infra-structures, the recent design concept trends a reliability-based design to consider many sources of uncertainties in structural design. However, researches on the impact factor of decks based on a probabilistic approach have not been advanced. Therefore, there is a need to fill this gap.

This paper reports a probabilistic assessment of code-specified impact factors for decks considering randomness of the influencing factors to dynamic responses of decks by means of a three-dimensional traffic-induced dynamic response analysis of bridges based on the modal analysis [9] combined with the Monte Carlo simulation (MCS) technique.

\section{Governing Equations of Bridge-Vehicle Interaction System}

The method called Lagrange equation of motion is adopted for the formulation of the governing equation of a bridge-vehicle interaction system as shown in Eq. (1).

$$
\frac{d}{d t}\left(\frac{\partial T}{\partial \dot{q}_{i}}\right)-\frac{\partial T}{\partial q_{i}}+\frac{\partial V}{\partial q_{i}}+\frac{\partial U_{d}}{\partial \dot{q}_{i}}=0
$$

where, $T, V$ and $U_{d}$ are the kinematic, potetial and dissipation energies of the interaction system, respectively. $q_{i}$ is the $i$-th generalized co-ordinate.

The kinematic, potential including strain energy and dissipation energies due to the viscous damping of the bridge-vehicle interaction system are expressed in a set of generalized coordinates as follows [9]. It 
is noteworthy that the superscript dot on variables denotes differential with respect to time.

$$
\begin{gathered}
T=\frac{1}{2}\left[\dot{\mathbf{D}}^{T} \mathbf{M}_{b} \dot{\mathbf{D}}+\sum_{v=1}^{n_{v e h}} T_{v}\right] \\
V=\frac{1}{2}\left[\mathbf{D}^{T} \mathbf{K}_{b} \mathbf{D}+\sum_{v=1}^{n_{v e h}} \sum_{m=1}^{3} \sum_{u=1}^{2} V_{v}\right] \\
U_{d}=\frac{1}{2}\left[\dot{\mathbf{D}}^{T} \mathbf{C}_{b} \dot{\mathbf{D}}+\sum_{v=1}^{n_{v e h}} \sum_{m=1}^{3} \sum_{u=1}^{2} U_{d v}\right]
\end{gathered}
$$

where,

$$
\begin{aligned}
& T_{v}=\sum_{k=1}^{2}\left(m_{v 1 k} \dot{Z}_{v 1 k}^{2}+J_{x v 1 k} \dot{\theta}_{x v 1 k}^{2}+J_{y v k k} \dot{\theta}_{y v k k}^{2}\right)+m_{v 22} \dot{Z}_{v 22}^{2}+J_{x v 22} \dot{\theta}_{x v 22}^{2} \\
& V_{v}=K_{v m 1 u} R_{v m 1 u}^{2}+K_{v m 2 u}\left(R_{v m 2 u}-Z_{0 v m u}\right)^{2}+2 W_{v m u} Z_{0 v m u} \\
& U_{d v}=C_{v m 1 u} \dot{R}_{v m 1 u}^{2}+C_{v m 2 u}\left(\dot{R}_{v m 2 u}-\dot{Z}_{0 v m u}\right)^{2} \\
& Z_{0 v m u}=w\left(t, x_{v m u}\right)-Z_{r v m u}
\end{aligned}
$$

$$
\begin{aligned}
& R_{v m k u}= \begin{cases}Z_{v 11}-(-1)^{m} \lambda_{x v m} \theta_{y v 11} & -(-1)^{u} \lambda_{y v 1} \theta_{x v 11}-Z_{v m 2} \\
& +(-1)^{u} \lambda_{y v(m+1)} \theta_{x v m 2} \\
& \text { for } m=1,2 ; k=1 ; u=1,2 \\
Z_{v 12}-(-1)^{u} \lambda_{y v 2} \theta_{x v 12} & \text { for } m=1 ; k=2 ; u=1,2 \\
0 & \text { otherwise }\end{cases} \\
& \left.W_{v m u}=\left\{\begin{array}{ll}
\frac{1}{2} g \\
\frac{1}{4} g\left[\left(1-\frac{\lambda_{x v 1}}{\lambda_{x v}}\right) m_{v 11}+m_{v 12}\right. \\
0
\end{array}\right] \begin{array}{l}
\text { for } \mathrm{m}=1 ; \mathrm{u}=1,2 \\
m_{x v 1}+m_{v 22}
\end{array}\right] \begin{array}{l}
\text { for } \mathrm{m}=2,3 ; \mathrm{u}=1,2 \\
\text { otherwise }
\end{array}
\end{aligned}
$$

In the equations, $J$ and $g$ indicate the mass moment of inertia of vehicles and gravity acceleration, respectively. $\mathbf{D}$ and $\dot{\mathbf{D}}$ indicate displacement and velocity vectors of a bridge, respectively; $\mathbf{M}_{b}$ and $\mathbf{K}_{b}$ respectively indicate mass and stiffness matrices of a bridge; $\mathrm{C}_{b}$, the damping matrix of a bridge derived from the assumption of a linear relation between the mass and stiffness matrices.

The symbols $Z_{v 11}, Z_{v 12}, Z_{v 22}, \theta_{x v 11}, \theta_{x v 12}, \theta_{x v 22}, \theta_{y v 11}$ and $\theta_{y v 22}$ refer to vehicle motions in relation to the bounce, parallel hop of the front axle, parallel hop of the rear axle, rolling, axle tramp of the front axle, axle tramp of the rear axle, pitching and axle windup of the rear axle, 


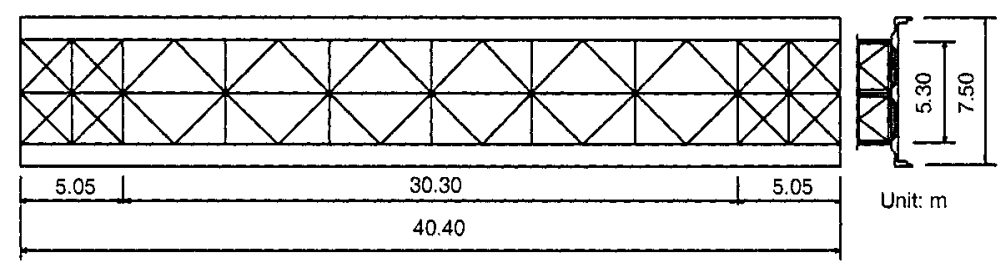

Figure 1. Plan view of bridge model

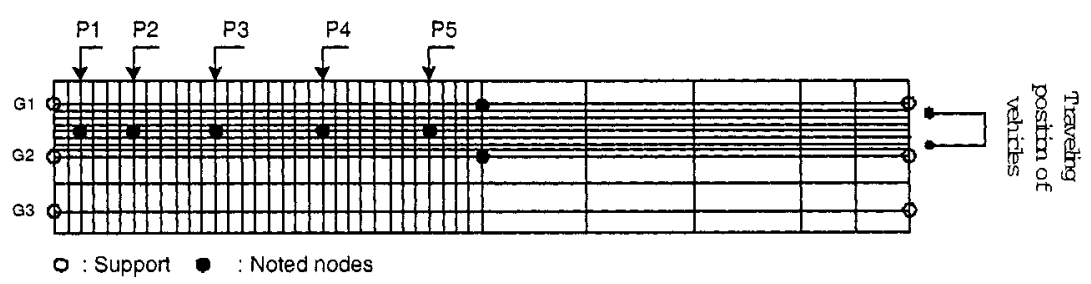

Figure 2. FE model of bridge

respectively. $m_{v 11}, m_{v 12}$ and $m_{v 22}$ indicate the concentrated mass on the vehicle body, front axle and rear axle, respectively.

$K_{v m k u}$ and $C_{v m k u}$ are the spring constant and damping coefficient of the $v$-th vehicle; the subscript $k$ is the index for indicating the vehicle body and axle ( $k=1$ : vehicle body; $k=2$ : axle), $m$ is the index for positions of axles or tires $(k=1$ and $m=1$ : front axle; $k=2$ and $m=1$ : rear axle; $k=2$ and $m=1$ : wheel at the front axle; $k=2$ and $m=2$ : front wheel at the tandem axle; $k=2$ and $m=3$ : rear wheel at the tandem axle) and $u$ is the index for indicating left and right sides of the $v$-th vehicle ( $u=1,2$ indicating left and right side, respectively).

The symbol $n_{v e h}$ means numbers of the vehicles on a bridge. The variable $z_{0 v m u}$ denotes the vehicle displacement from the datum before deformation to a wheel after deformation of a bridge including roadway roughness. The longitudinal position of a wheel location $x_{v m u}$ is relative to the bridge entrance. The pavement roughness of the bridge at a wheel is denoted by $z_{r v m u}$. The variable $w\left(t, x_{v m u}\right)$ is the elastic deformation of the bridge at a location of $x_{v m u}$ and a time of $t$. The subscript $v$ indicates the $v$-th vehicle on the bridge.

The final formulation of governing differential equations for a bridgevehicle interaction system is obtained from the relations in Eq. (1) to Eq. (4). 
Table 1. Property of steel bridge

\begin{tabular}{lr}
\hline Mass per unit length $(\mathrm{kg} / \mathrm{m})$ & 7550.000 \\
Sectional area of girders $\left(\mathrm{m}^{2}\right)$ & 0.142 \\
Moment of inertia $\left(\mathrm{m}^{4}\right)$ & 0.212 \\
Torsional constant $\left(\mathrm{m}^{4}\right)$ & 0.055 \\
Damping constants for the 1st and 2nd modes & 0.025 \\
\hline Fundamental frequency & 1st: bending \\
(Experiment, $\mathrm{Hz}$ ) & 2.340 \\
\hline
\end{tabular}

\section{Model Description}

\subsection{Bridge Model}

A simple span bridge considered is a steel composite plate-girder bridge with span length of $40.4 \mathrm{~m}$, and composed with three girders. The span length and thickness of the $\mathrm{RC}$ deck are $2.65 \mathrm{~m}$ and $17 \mathrm{~cm}$, respectively. Table 1 shows the properties of the bridge used in the dynamic response analysis. The fundamental frequencies for the bending and torsional modes taken from the eigenvalue analysis are calibrated to coincide with experimental values obtained from field-test data. Validity of the analytical responses is verified by comparing with field-test data [8].

The plan view and finite element model of the bridge are shown in Figure 1 and Figure 2, respectively. Analyzed panels are denoted as P1, P2, P3, P4 and P5 as shown in Figure 2. The FE model consists of 494 nodes, 444 flat shell elements and 223 beam elemts. The response of decks is calculated by superposing up to the 330 th mode that coincides with the 5th bending mode of the deck with frequency of about $750 \mathrm{~Hz}$, since the dynamic responses are sufficiently converged within the 330th mode from a preliminary analysis.

\section{$2.2 \quad$ Vehicle Model}

Traffics with high percentage of heavy trucks on highway bridges usually occur at night, and the maximum traffic constitution among heavy trucks has been reported as the three-axle vehicle [14]. Moreover, Kim and Kawatani [12] demonstrate that the three-axle dump truck with a rear tandem axle gives rise to the maximum impact factor at the decks near expansion joints due to bumps. Thus, a dump truck with a tandem axle idealized as an eight-degree-of-freedom model is adopted as a vehicle model [9]. Properties of the vehicle model are summarized in Table 2. 
Table 2. Property of vehicle

\begin{tabular}{clr}
\hline Geometry $(m)$ & Tread & 1.80 \\
& Distance between front and rear axles & 3.99 \\
& Distance of tandem axle & 1.32 \\
& Distance between front axle and C.G. & 2.99 \\
\hline Weight $(k N)$ & Gross & 191.00 \\
& Sprung mass including payload & 171.00 \\
& Steer axle: unsprung mass & 4.90 \\
& Drive axle: unsprung mass & 14.70 \\
\hline Spring constant & Front leaf spring & 1577.00 \\
$(k N / m)$ & Rear leaf spring & 4724.00 \\
& Front tire & 3146.00 \\
& Rear tire & 4724.00 \\
\hline Damping coefficient & Front suspension & 4.60 \\
$(k N \cdot s / m)$ & Rear suspension & 13.72 \\
& Front tire & 9.11 \\
& Rear tire & 27.34 \\
\hline Fundamental frequency & Bounce & 3.00 \\
$(H z)$ & Parallel hop & 17.90 \\
\hline C.G: Centre of gravity &
\end{tabular}

\subsection{Random Variables}

As random variables that effect on the dynamic response of decks, the roadway roughness on the bridge surface, bump height at the expansion joint of the bridge entrance, traveling position of vehicles, vehicle running speed and axle load at each axle of dump trucks are considered.

Roadway Profile. The fluctuations of roadway surface can be treated as a homogeneous, Gaussian random process with zero mean [4]. The simplest model describes the roadway surface as a cylindrical surface defined by a single longitudinal profile $z_{r}(x)$. Assuming $z_{r}(x)$ to be a zero mean, homogeneous, Gaussian random process as shown in Eq. (5), its probability structure can be defined by the auto-correlation function, or by the power spectral density(PSD).

$$
z_{r}(x)=\sum_{k=1}^{M} a_{k} \sin \left(\omega_{k} x+\varphi_{k}\right)
$$

where, $\alpha_{k}$ is Gaussian random variable with zero mean and variance $\sigma_{k}^{2}=4 S\left(\omega_{k}\right) \Delta \omega, \varphi_{k}$ is a random variable having uniform distribution between 0 and $2 \pi, \omega_{k}$ is the circular frequency of roadway surface roughness written as $\omega_{k}=\omega_{L}+(k-1 / 2) \Delta \omega, \Delta \omega=\left(\omega_{U}-\omega_{L}\right) / M, \omega_{U}$ and 
$\omega_{L}$ designate the upper and lower limit of the frequency, respectively, $M$ means a large enough integer number and $S\left(\omega_{k}\right)$ is the PSD of a roadway profile.

The PSD can be obtained by a spectral analysis of the roadway profile measured along any longitudinal section. Following analytical description has been proposed to fit the measured PSD [7].

$$
S(\Omega)=\frac{\alpha}{\Omega^{n}+\beta^{n}}
$$

where, $\alpha$ is roughness coefficient, $\Omega(=\omega / 2 \pi)$ is space frequency (cy$\mathrm{cle} / \mathrm{m}), \beta$ designates shape parameter and $n$ means parameter to express the distribution of power of the PSD curve.

If a PSD for a roadway profile is defined, then, by means of the MCS method, samples of roadway profiles can be obtained using the sampling function shown in Eq. (5). As parameters in Eq. (6), $\alpha=0.001$, $\beta=0.05$ and $n=2.0$ are used in this study based on measured data of Meishin Expressway in Japan [10]. The roadway roughness condition can be categorized as the road class "A" corresponds to a very good road according ISO 8086 code [2], which typically indicates a newly paved highway.

Bump Height near Expansion Joints. The extreme Type I distribution is assumed to describe bump heights at expansion joints of bridges based on the surveying results of national roadways in Japan [6]. Among the shapes of the measured bump profiles, the sine shaped bump profile that gives the most severe effect on the impact factors of decks from a preliminary study is adopted in the simulation. Although the mean value and standard deviation of the measured bump heights on the national roadways are $20.4 \mathrm{~mm}$ and $7.0 \mathrm{~mm}$, respectively, a half of the measured height is considered in the analysis for highway bridges [6]. The cumulative distribution function (CDF) and probability density function $(\mathrm{PDF})$ for the random variables are

$$
\begin{gathered}
F_{x}(x)=e^{-e^{-\alpha(x-u)}} \\
f_{x}(x)=\alpha e^{-e^{-\alpha(x-u)}} e^{-\alpha(x-u)}
\end{gathered}
$$

where, $u$ and $\alpha$ are distribution parameters; $\alpha \approx 1.282 / \sigma_{x}, u \approx \mu_{x}-$ $0.45 \sigma_{x}$ [3]. The $\sigma_{x}$ and $\mu_{x}$ indicate the standard deviation and mean value of a random variable $x$. 
Traffic Data. The normal distribution is assumed for the running speed and traveling position of vehicles on highway bridges based on the database of Hanshin Expressway. The PDF of the normal distribution for a normal random variables $x$ is shown in Eq. (9). The CDF of the normal random variables can be expressed as Eq. (10), even though there is no closed-form solution for the CDF of a normal random variable. The mean value and standard deviation of vehicle speeds are assumed as $70 \mathrm{~km} / \mathrm{hr}$ and $10 \mathrm{~km} / \mathrm{hr}$, respectively [14]. Those mean value and standard deviation for the traveling position of vehicles are $0.0 \mathrm{~m}$ and $0.2 m$ from a target passage [13].

$$
\begin{gathered}
f_{x}(x)=\frac{1}{\sigma_{x} \sqrt{2 \pi}} \exp \left[-\frac{1}{2}\left\{\frac{x-\mu_{x}}{\sigma_{x}}\right\}^{2}\right] \\
F_{x}(x)=\Phi\left(\frac{x-\mu_{x}}{\sigma_{x}}\right)
\end{gathered}
$$

The lognormal distribution is assumed for the axle load of the threeaxle vehicles based on the measured data of Hanshin Expressway. The mean value and standard deviation for the axle loads are $49.805 \mathrm{kN}$ and $12.056 k N$ for the front axle, $90.507 k N$ and $34.276 k N$ for the front wheel of the tandem axle and $67.571 \mathrm{kN}$ and $31.637 \mathrm{kN}$ for rear wheel of the tandem axle [13]. The CDF and PDF for a lognormal random variable can be obtained by substituting $\ln (x), \mu_{\ln (x)}$ and $\sigma_{\ln (x)}$ into the Eq.(9) and Eq.(10) instead of $x, \mu_{x}$ and $\sigma_{x}$. It is noteworthy that the spring constants of vehicles are rearranged to have natural frequencies of $3.0 \mathrm{~Hz}$ for the bounce and $17.9 \mathrm{~Hz}$ for the axle hop motion according to each sample of axle loads.

\section{Simulation of Impact Factor}

\subsection{Simulation and Probabilistic Feature}

A number of sample roadway profiles, bump heights, vehicle speeds, traveling positions of vehicles and axle loads are generated by means of the MCS method. Impact factors of each deck are analyzed according to each sample of the random variables by means of traffic-induced dynamic response analysis of bridges [9]. In the simulation, no correlation among the considered random variables is assumed. A hundred samples of the simulated random variables are considered in the analysis, since the simulated impact factors tend to converge within 100 samples in a preliminary study. 


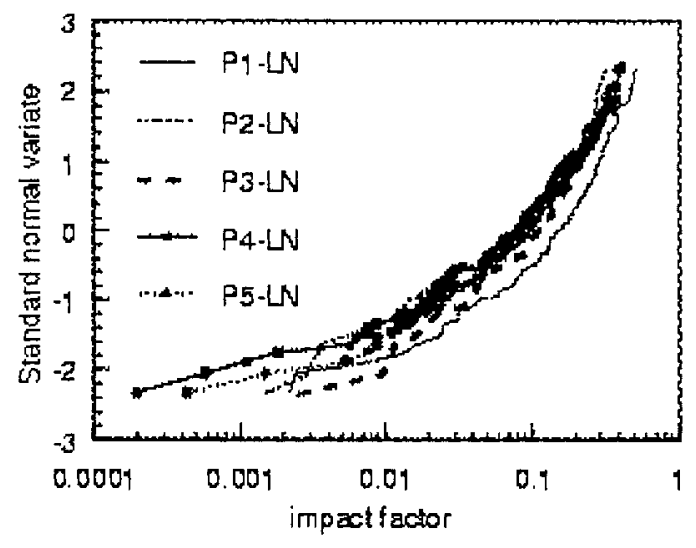

Figure 3. CDF of simulated impact factors of decks on lognormal probability paper

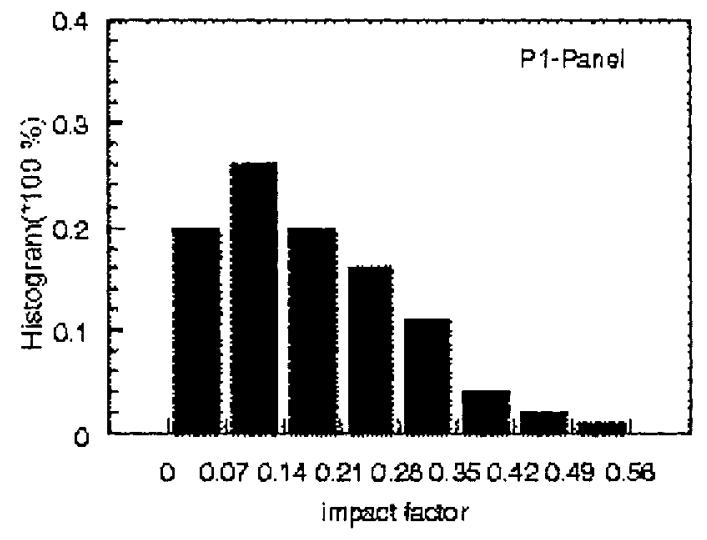

Figure 4. Distribution of simulated impact factors of P1-panel

Three types of distributions, such as normal, lognormal and extreme Type I distributions, are considered to investigate the probabilistic property of the simulated RC deck's impact factor. The CDF and histogram demonstrate that the impact factor can be concluded to follow lognormal distribution. Moreover the probability exceeding the code specified impact factors taken from the assumption of following the lognormal distribution gives the most frequent occurence among the three distributions. The CDF of the simulated impact factors plotted on lognormal probability paper is shown in Figure 3. The histogram of the simulated impact factors for the P1-panel is appeared in Figure 4. 


\subsection{Reliability of Code-Specified Impact Factors}

Table 3. Code specified impact factors for deck slab

\begin{tabular}{lll}
\hline Code & impact factor & $i_{\text {code }}$ \\
\hline AASHTO (USA) & $i=50 /(3.3 L+125) \leq 0.3$ & 0.300 \\
DIN1072 (Germany) & $i=0.4-0.008 L$ & 0.379 \\
JSHB (Japan) & $i=20 /(L+50)$ & 0.380 \\
OHBDC (Ontario, Canada) & $i=0.4$ & 0.400 \\
\hline
\end{tabular}

$L=2.65 \mathrm{~m}:$ Span length in meter

Table 4. Probability exceeding code specified impact factor (\%)

\begin{tabular}{c|ccccc}
\hline & P1 & P2 & P3 & P4 & P5 \\
\hline AASHTO & $11.15(3.77)$ & $3.16(3.85)$ & $4.25(5.20)$ & $4.08(3.27)$ & $3.58(3.35)$ \\
DIN1072 & $5.42(1.79)$ & $1.42(1.88)$ & $1.83(2.51)$ & $2.03(1.48)$ & $1.66(1.61)$ \\
JSHB & $5.37(1.77)$ & $1.40(1.87)$ & $1.82(2.47)$ & $2.01(1.47)$ & $1.64(1.59)$ \\
OHBDC & $4.50(1.49)$ & $1.56(1.58)$ & $1.49(2.09)$ & $1.70(1.21)$ & $1.37(1.34)$ \\
\hline
\end{tabular}

The reliability of the impact factors specified in AASHTO standard (USA), DIN1072 (Germany), Japanese Specifications of Highway Bridges (JSHB: Japan) and Ontario Highway Bridge Design Code (OHBDC Ontario, Canada) is investigated. The impact factors specified in the codes are summarized in Table 3.

The probability of exceeding the code-specified impact factors under the assumption of following lognormal distribution is summarized in Table 4. The value in the parenthesis indicates the exceeding probability against the code-specified impact factor without considering the bump at the expansion joint. Table 4 shows that the probability exceeding the code-specified impact factor for the deck near the bump is about three times greater than that of other decks.

The reliability index (RI) that calculated from the inverse of the exceeding probability is summarized in Figure 5 to compare the limit states defined in the EUROCODE [1]. The target reliability indices proposed in Eurocode are 1.5 for serviceability limit state (SLS), 3.8 for the ultimate limit state (ULS) and 1.5 to 3.8 for the fatigue limit state (FLS). The symbols - NB and -B indicate the results without considering bumps and with consdiering bumps at the expansion joint, respectively.

If the impact factor can be classified in the serviceability limit state then RI of the impact factor of AASHTO for the P1 panel is lower than that of the target reliability index for the SLS, although what kind of 


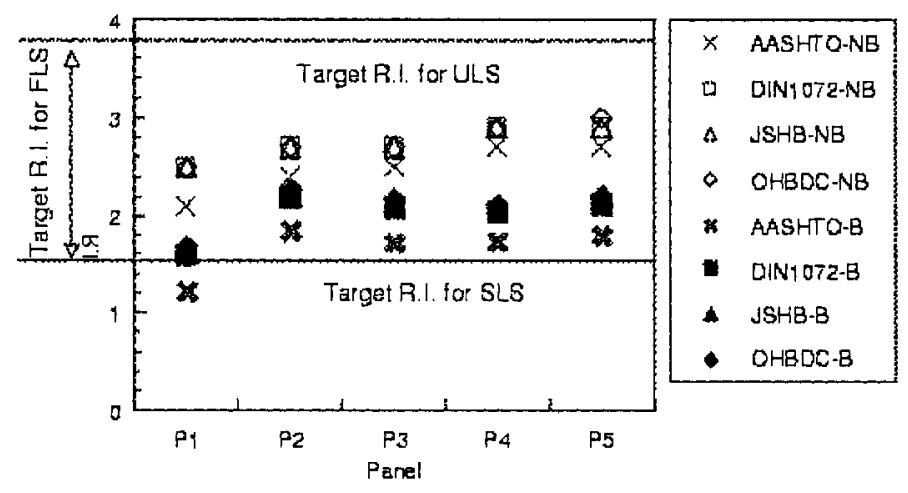

Figure 5. Reliability index of code-specified impact factors with target reliability index

limit state the impact factor is classified in has not been defined yet. On the other hand the reliability considering the condition of no bump at the expansion joint satisfies the SLS. It indicates that the bump is one of important factors for the impact factor of decks.

\section{Concluding Remarks}

The probabilistic feature of RC decks' simulated impact factors are examined. The reliability evaluation of code-specified impact factors is carried out considering randomness of the roadway roughness, bump height, vehicle speed, traveling position of vehicles and axle load. The study shows that the straight lines on the lognormal distribution paper can approximately represent probabilistic properties of the impact factor for the RC deck slab. The impact factor of the deck near expansion joints dominates the design impact factor. Therefore, if the impact factor of the deck near an expansion joint of an approaching side of bridges satisfies a given reliability due to a vehicle with tandem axle running on a bump, those reliabilities of other decks are satisfied automatically. In considering the impact factor of decks of bridges on roadways that have more severe bump condition than highway bridges, the reliability index against codes can decrease, therefore the use of code-specified impact factors of bridges on national roadways may overestimate the performance level of decks.

\section{References}

[1] Eurocode 1. Basis of design and action on structures, Part 1: Basis of design, 1993. Sixth draft. 
[2] ISO 8606. Mechanical Vibration - Road Surface Profiles - Reporting of Measured Data, 1995; British Standard, BS 7853, 1996.

[3] J. R. Benjamin and C. A. Cornell. Probability, Statistics and Decision for Civil Engineers. McGraw-Hill, New York, 1970.

[4] C. J. Dodds and M. M. Robson. The description of road surface roughness. Int. J. Sound and Vibrations, 31(2):175-183, 1973.

[5] H. Furuta, I. Tsukiyama, M. Dogaki, and D. M. Frangopol. Maintenance support system of steel bridges based on life cycle cost and performance evaluation. Proceedings of the 10th IFIP WG7.5 Working Conference on Reliability and Optimization of Structural Systems, pages 205-213, 2002.

[6] H. Honda, Y. Kajikawa, and T. Kobori. Roughness characteristics at expansion joint on highway bridges. Proceedings of JSCE, Note, 328:173-176, 1982. (in Japanese).

[7] H. Honda, Y. Kajikawa, and T. Kobori. Spectra of road surface roughness on bridges. ASCE Structural Division, 1081(ST9):1956-1966, 1982.

[8] M. Kawatani and C. W. Kim. Effects of gap at expansion joint on traffic-induced vibration of highway bridge. Proc. Int. Conference on Developments in Short and Medium Span Bridge Engineering '98, CD-ROM, 1998.

[9] M. Kawatani and C. W. Kim. Computer simulation for dynamic wheel loads of heavy vehicles. Int. J. Structural Engineering and Mechanics, 12(4):409-428, 2001.

[10] M. Kawatani, Y. Kobayashi, and K. Takamori. Nonstationary random analysis with coupling vibration of bending and torsion of simple girder bridges under moving vehicles. JSCE, J. Structural Eng. and Earthquake Eng., 15(1):107s$114 \mathrm{~s}, 1998$.

[11] C. W. Kim and M. Kawatani. A probabilistic investigation on impact factor of deck slabs of highway bridges. Proc. of the 9th IFIP WG7.5 Working Conference on Reliability and Optimization of Structural Systems, pages 125-133, 2000.

[12] C. W. Kim and M. Kawatani. Probabilistic investigation on impact factor of deck slabs due to truck configuration type. Proceedings of the 10th IFIP WG7.5 Working Conference on Reliability and Optimization of Structural Systems, pages 87-94, 2002.

[13] Comittee of Technology on Concrete of HEPC. Crack damage of RC deck slabs of highway bridges and its resistance. Technical report, Hanshin Expressway Management Technology Center, 1991. (in Japanese).

[14] M. Sakano, I. Mikami, and K. Miyagawa. Simultaneous loading effect of plural vehicles on fatigue damages of highway bridges. IFIP Transactions B-12, Reliability and Optimization of Structural Systems, $V$, pages 221-228, 1993.

[15] K. Yokoyama, J. Inoue, and T. Nagahara. Field test on the impact coefficient of steel deck and reinforced concrete slab of highway bridges. JSCE, J. of Structural Engineering, 35A:749-756, 1989. (in Japanese). 